\title{
Frecuencia de erliquiosis y anaplasmosis en perros con historial de garrapatas en una clínica veterinaria de Piura, Perú
}

\author{
Frequency of ehrlichiosis and anaplasmosis in dogs with a history of ticks in a veterinary clinic in Piura, \\ Peru
}

\author{
Natalia Naranjo ${ }^{1}$, Renato Zúñiga ${ }^{2}$
}

\section{RESUMEN}

El objetivo del estudio fue determinar la frecuencia de serología positiva para las enfermedades de erliquiosis $(E$. canis y E. ewingii) y anaplasmosis (A. phagocitophylum y A. platys) en perros con presencia o historial de garrapatas en la ciudad de Piura. Durante el periodo de 2017-2018, se seleccionaron perros que llegaron a consulta a una clínica veterinaria privada en la ciudad de Piura - Perú. El principal criterio de inclusión fue que debían tener presencia de garrapatas o antecedentes de haberlas presentado según los dueños. Se registró mediante una ficha, los signos que presentaron al momento de la consulta y que fueran compatibles a las enfermedades en estudio. Se recolectaron muestras de 71 individuos para ser analizadas mediante hemograma y una prueba comercial para la detección serológica de estas enfermedades (SNAP 4Dx plus - IDEXX). Se determinó que el 55\% (39) de pacientes presentaron anticuerpos de Erlichia spp. y 4\% (3) contra Anaplasma spp. Todos los individuos que poseían anticuerpos contra Anaplasma spp., también poseían anticuerpos contra Erlichia spp. Entre los signos clínicos encontrados con mayor frecuencia en el grupo de positivos fueron letargia (77\%), anorexia (59\%), linfoadenomegalia (54\%) y pirexia (49\%), sin llegar a ser significativamente diferentes a los negativos. El único signo con mayor frecuencia con respecto al grupo de negativos fue la esplenomegalia ( $49 \%$ en el grupo de positivos y $6 \%$ en el grupo de negativos). Se determinó que los individuos con serología positiva tenían como alteraciones hematológicas principales: anemia (51\%), leucocitosis (51\%) y trombocitopenia (54\%), mientras que los negativos sólo obtuvieron alteraciones en la serie blanca. Este estudio reporta por primera vez la presencia serológica de Anaplasma sp. y corrobora la presencia de Erlichia spp. en la ciudad de Piura.

PALABRAS CLAVE: Ehrlichia spp., Anaplasma sp., Piura, serología.

\section{SUMMARY}

The objective of the study was to determine the frequency of positive serology for canine erlichiosis (E. canis and E. ewingii) and anaplasmosis (A. phagocitophylum and A. platys) diseases in patients with presence or history of ticks during the period 2017-2018. The sample was composed of dogs who came to consultation to a private veterinary service in the city of Piura-Peru. These patients must have had presence or history of ticks, according to the owners. The information of the clinical signs that were presented at the time of the consultation and that could be compatible with the disease under study were collected in a medical card. Samples of 71 dogs were collected to be analyzed by blood count and a commercial test for the detection of these diseases (SNAP 4Dx- Idexx). It was determined that 55\% (39 patients) presented antibodies against Erlichia spp. and 4\% (3 patients) Anaplasma sp. All of the patients who presented antibodies against Anaplasma sp. also presented antibodies against Erlichia spp. Among the clinical

Clínica Veterinaria "Santa María del Pinar". Piura, Perú.

Laboratorio de análisis clínico Vet Support. Lima, Perú. 
signs found more frequently in the positive group were lethargy (77\%), anorexy (59\%), linfoadenomegaly (54\%) and fever (49\%), without becoming significantly different from the negatives. The only sign more frequent among the negative group was the splenomegaly ( $49 \%$ positive group and $6 \%$ negative group). It was determined that the patients with positive serology had alterations in their blood count like anemia (51\%), leukocytosis (51\%) and thrombocytopenia (54\%), while the negative group only got alterations in the white cells. This study reports the serological presence of Anaplasma sp. for the first time and corroborates the presence of Erlichia spp. in the city of Piura.

KEY WORDS: Ehrlichia spp., Anaplasma sp., Piura, serology.

\section{INTRODUCCIÓN}

Las enfermedades infecciosas transmitidas por vectores en perros son un grupo importante de enfermedades debido a que muchas de ellas son zoonóticas. Durante los últimos años, la incidencia de estas enfermedades han ido en aumento en varios países tropicales y subtropicales, así como en países de zonas templadas (Miró et al., 2013). Entre los factores reconocidos en este incremento se encuentran los cambios en la dinámica de la población de perros y los cambios medio ambientales (Dantas-Torres, 2008). La erliquiosis y anaplasmosis son producidas por organismos intracelulares pertenecientes al orden Rickettsiales, familia Anaplasmataceae, géneros Ehrlichia spp. y Anaplasma spp., respectivamente (Dolz et al., 2013).

La erliquiosis o rickettsiosis monocítica que afecta a los perros, es producida por la bacteria Ehrlichia canis, que se transmite principalmente por medio del artrópodo de la familia Ixodidadae, Rhipicephalus sanguineus o también llamada garrapata marrón del perro (Trapp et al., 2006; Romero, 2011; Huerta y Dámaso, 2015). El periodo de incubación de la enfermedad es de 8 a 20 días, durante este tiempo los microorganismos infectan y se multiplican en los macrófagos y el sistema fagocítico mononuclear, por medio de fisión binaria y se diseminan por todo el organismo del animal (Baneth, 2006; Romero, 2011).

La erliquiosis canina se detectó en 1982 por primera vez en el Perú (Chavera et al., 1982), y posteriormente se ha ido reportando en distintos lugares de nuestro país (Adrianzén et al., 2003). En la ciudad de Sullana, Piura se ha reportado un $76 \%$ de seropositividad (San Miguel, 2006).

La anaplasmosis canina es una enfermedad causada por agentes bacterianos intracelulares como Anaplasma phagocytophilum y Anaplasma platys (Alleman \& Wamsley, 2008). Al igual que la erliquiosis, la anaplasmosis también es transmitida por la garrapata marrón del perro y otros vectores (Riphicephalus spp. e Ixodes spp) (Rubio et al., 2011; Tateishi et al., 2015). La infección por $A$. phagocytophilum afecta frecuentemente a las células blancas de tipo polimorfonuclear, entre ellas los neutrófilos; mientras que $A$. platys produce una infección intracelular de plaquetas ocasionando la trombocitopenia cíclica infecciosa del perro (Greene, 2012).

La temperatura ambiental es uno de los factores más influyentes en el ciclo de vida de las garrapatas, debido a que durante la primavera y verano, que son los meses de mayor temperatura, estas logran alimentarse y reproducirse con mayor rapidez (Manzano et al., 2012). La ciudad de Piura, Perú posee estas condiciones, y por acercarse a la línea ecuatorial, alcanza una temperatura promedio de $17-33^{\circ} \mathrm{C}$. Esta condición la hace propicia para la proliferación de estos artrópodos, y es por ello que son endémicas de climas tropicales y subtropicales (Dantas-Torres, 2008). En esta provincia existen antecedentes de infecciones por erliquiosis y dado que comparten el mismo vector, es probable que la anaplasmosis esté afectando también a los perros. Por ello, el objetivo del estudio fue determinar la frecuencia de exposición por Erlichia spp. y Anaplasma sp., en perros que acudieron a un servicio veterinario particular de la provincia de Piura.

\section{MATERIAL Y METODOS}

La investigación se realizó en la Clínica Veterinaria "Santa María del Pinar" ubicada en el distrito, provincia y departamento de Piura. El estudio se desarrolló durante los meses octubre 2017 a marzo 2018. La población objetivo fueron perros sin distinción de raza, sexo o edad que se presentaron a consulta, con historial y/o presencia de garrapatas.

El número de muestra se definió mediante la fórmula de comprobación de una proporción. Se 
utilizó una prevalencia referencial de Erlichia spp. de $76 \%$ (San Miguel, 2006), un nivel de confianza del $95 \%$ y un error máximo admisible del 10\%. El tamaño de muestra calculado fue de 71 perros.

La información de los pacientes fue recogida a través de una ficha en la cual se consideraron las siguientes variables: sexo, raza, permanencia en casa, procedencia, edad, presencia de garrapatas, historia clínica y hallazgos al examen clínico. La obtención de la muestra de sangre requirió la sujeción de los pacientes, la cual fue realizada por personal de apoyo de la clínica veterinaria siguiendo los procedimientos convencionales (Gordillo, 2010). La muestra se traspasó a tubos con EDTA de 1-3ml siendo homogenizadas de manera inmediata mediante inversión unas 10 veces, luego del cual fueron etiquetadas e identificadas con el nombre y código asignado a cada paciente. Si las muestras no iban a ser procesadas dentro de los siguientes 15 minutos se procedió a mantenerlas en temperatura de refrigeración a $4^{\circ} \mathrm{C}$. Todas las muestras fueron procesadas dentro de las 12 horas post extracción.

Las muestras fueron utilizadas para realizar el hemograma con el equipo hematológico IDEXX
VetAutoread $\AA$, frotis sanguíneo y la prueba serológica 4 Dx IDEXX ${ }^{\circledR}$, bajo el procedimiento estándar proporcionado por la misma marca. Los resultados del SNAP se interpretaron a los 8 minutos después de realizada la prueba, y se interpretó como resultado positivo cuando se observó color en el punto de control (esquina superior izquierda) y cualquier indicio de color en punto que indicaba Erlichia spp. (punto ubicado a la izquierda) o el punto que indicaba Anaplasma $s p$. (punto superior); se tomó como resultado negativo cuando sólo apareció el punto control (IDEXX, 2008). La preparación del frotis sanguíneo y su lectura siguió protocolos de laboratorio establecidos (Pérez et al., 2011). La observación de la lámina incluyó el contaje e identificación de 100 leucocitos siguiendo la técnica del Zigzag de Shiling, evaluación de la morfología de los eritrocitos leucocitos y la presencia de cuerpos de inclusión intra/extracelulares. El recuento de plaquetas utilizó el mismo frotis teñido que se usó para el recuento diferencial de leucocitos siguiendo procedimientos convencionales (Cowell et al, 1999).

Los resultados fueron resumidos a través de estadística descriptiva y presentados en tablas. Se determinó la asociación entre serología positiva con la permanencia en casa y signos mediante la prueba

Tabla 1. Presencia de anticuerpos contra Erlichia spp y Anaplasma sp. en caninos atendidos en un servicio veterinario privado de Piura durante el período primavera 2017 - verano 2018.

\begin{tabular}{|c|c|c|c|c|c|}
\hline \multirow{2}{*}{ Variable } & \multirow{2}{*}{ Muestra } & \multicolumn{2}{|c|}{ Erlichia spp. (+) } & \multicolumn{2}{|c|}{ Anaplasma sp. (+) } \\
\hline & & $\mathbf{n}$ & $\%$ & $\mathbf{n}$ & $\%$ \\
\hline \multicolumn{6}{|l|}{ Sexo } \\
\hline Macho & 44 & 26 & 59,1 & 3 & 6,8 \\
\hline Hembra & 27 & 13 & 48,1 & 0 & 0,0 \\
\hline \multicolumn{6}{|l|}{ Distrito } \\
\hline Piura & 52 & 23 & 44,2 & 1 & 1,9 \\
\hline Otros distritos* & 19 & 16 & 84,2 & 2 & 10,5 \\
\hline \multicolumn{6}{|c|}{ Presencia de garrapatas } \\
\hline $\mathrm{Si}$ & 61 & 35 & 57,4 & 3 & 4,9 \\
\hline No & 10 & 4 & 40,0 & 0 & 0,0 \\
\hline \multicolumn{6}{|l|}{ Raza } \\
\hline No definida & 12 & 9 & 75,0 & 1 & 8,3 \\
\hline Definida & 59 & 30 & 50,8 & 2 & 3,4 \\
\hline \multicolumn{6}{|c|}{ Permanencia en casa } \\
\hline Temporal & 36 & 26 & $72,2^{\mathrm{b}}$ & 2 & 5,5 \\
\hline Nula & 2 & 1 & $50,0^{\mathrm{b}}$ & 0 & 0.0 \\
\hline Exclusiva & 33 & 12 & $36,4^{\mathrm{a}}$ & 1 & 3,0 \\
\hline
\end{tabular}


de Chi Cuadrado; además se utilizó la prueba T de Student para comparar las medias de los valores hematológicos de los individuos positivos y negativos a la prueba.

Los procedimientos fueron realizados con la aprobación del Comité de Ética de la Universidad Peruana Cayetano Heredia por medio de la constancia 067-07-17. Los propietarios confirmaron su participación en el estudio con la firma de un consentimiento informado.

\section{RESULTADOS}

El estudio recuperó 71 muestras de perros con presencia de garrapatas $\mathrm{y} / \mathrm{o}$ historial reciente de estas y con signos compatibles de las enfermedades transmitidas por vectores, que llegaron a consulta a la Clínica Veterinaria "Santa María del Pinar" sede del estudio.

La seropositividad de Erlichia spp. fue de 55\% $(\mathrm{n}=39)$ y la de Anaplasma sp. de 4.2\% $(\mathrm{n}=3) . \mathrm{El}$

Tabla 2. Signos clínicos presentados en animales positivos y negativos a Ehrlichia spp., atendidos en un servicio veterinario privado de Piura durante el período primavera 2017 - verano 2018.

\begin{tabular}{lcccc}
\hline \multicolumn{1}{c}{ Signos clínicos } & \multicolumn{2}{c}{ Canes positivos $(\mathbf{n = 3 9 )}$} & \multicolumn{2}{c}{ Canes Negativos (n=32) } \\
& $\mathbf{n}$ & $\mathbf{\%}$ & $\mathbf{n}$ & $\mathbf{\%}$ \\
\hline Vómitos & 11 & 28,2 & 15 & 46,9 \\
Diarrea & 10 & 25,6 & 7 & 21,9 \\
Anorexia & 23 & 59 & 19 & 59,4 \\
Sangrado & 12 & 30,8 & 4 & 12,5 \\
Cojeras & 15 & 38,5 & 10 & 31,3 \\
Pirexia & 19 & 48,7 & 12 & 37,5 \\
Petequias & 7 & 17,9 & 4 & 12,5 \\
Letargia & 30 & 76,9 & 20 & 62,5 \\
Linfoadenomegalia & 21 & 53,8 & 12 & 37,5 \\
Esplenomegalia & 19 & $48,7^{\mathrm{a}}$ & 2 & $6,3^{\mathrm{b}}$ \\
Descarga oculonasal & 9 & 23,1 & 11 & 34,4 \\
Reflejo Tusígeno & 13 & 33,3 & 15 & 46,9 \\
\hline
\end{tabular}

${ }^{\text {a.b }}$ Letras diferentes indican que las proporciones son diferentes estadísticamente $(\mathrm{p}<0.05)$

Tabla 3. Valores hematológicos en pacientes canes positivos y negativos a Ehrlichia spp., atendidos en un servicio veterinario privado de Piura durante el período primavera 2017 - verano 2018.

\begin{tabular}{lccc}
\hline Hemograma & Rango de referencia & $\begin{array}{c}\text { Erliquiosis (+) } \\
\text { mínimo - } \\
\text { máximo }\end{array}$ & $\begin{array}{c}\text { Erliquiosis (-) } \\
\text { mínimo - máximo }\end{array}$ \\
\hline Hemoglobina & $12-17 \mathrm{~g} / \mathrm{dl}$ & $2,3-19,3$ & $4,6-18,8$ \\
Hematocrito* & $37-50$ & $8-54,7$ & $14,2-53,9$ \\
CHCM & $31-37 \mathrm{~g} / \mathrm{dl}$ & $28,7-36,5$ & $30,2-35,3$ \\
Leucocitos & $9000-15000 / \mathrm{ul}$ & $5000-59700$ & $1800-45300$ \\
Neutrófilos & $5400-11550 / \mathrm{ul}$ & $3900-47163$ & $1029-42129$ \\
Linfocitos & $1350-4500 / \mathrm{ul}$ & $103-12760$ & $168-5750$ \\
Basófilos & $0-150 / \mathrm{ul}$ & 0 & 0 \\
Monocitos & $180-450 / \mathrm{ul}$ & $148-7164$ & $76-1764$ \\
Plaquetas & $200000-500000 / \mathrm{ul}$ & $4000-565000$ & $33000-756000$ \\
\hline *Indica que las comparaciones de las medias son significativamente diferentes para la variable \\
evaluada (p<0.05)
\end{tabular}


detalle de la distribución de los resultados distribuidos según variables de estudio se presenta en la tabla 1 . Se realizó estadística únicamente para los animales positivos a erliquiosis ya que el número de pacientes con anaplasmosis fue bajo. Se encontró diferencia significativa entre la proporción de positivos a Erlichia spp. y la permanencia temporal de los perros en las viviendas, siendo menor en el caso de los animales confinados todo el tiempo.

El $70 \%$ de los individuos evaluados llegaron a consulta por letargia y/o decaimiento. La tabla 2 muestra los signos clínicos observados en consulta, así como los signos observados por los dueños en animales positivos y negativos a Erlichia spp. Solo se encontró diferencia significancia para la variable presencia de esplenomegalia, siendo la frecuencia mayor en individuos positivos a Erlichia spp. $(\mathrm{p}<0.05)$.

Los resultados de los valores hematológicos encontrados en los individuos con serología positiva y negativa a Erlichia spp. se presenta en la tabla 3. Se halló una asociación significativa entre la seropositividad Erlichia spp. y la variable hematocrito. Adicionalmente, en la observación de los frotices sanguíneos de los individuos evaluados, se hallaron 2 cuerpos de inclusión de distemper y 2 cuerpos de inclusión de Erlichia spp.

\section{DISCUSIÓN}

Los resultados del estudio cuantifican una seropositividad total para Erlichia spp. de 55\%. A pesar de encontrar un alto porcentaje de seropositividad en la población de perros; este resultado fue menor al encontrado por un estudio previo realizado en el departamento de Piura (San Miguel, 2006). Esto podría estar relacionado a que el estudio actual solo evaluó a perros que tenían dueño, más no perros callejeros, cuya inclusión incrementaría el riesgo de contagio para la enfermedad y aumentaría los casos positivos..

Considerando la variable sexo, se obtuvo que $62 \%$ de los pacientes fueron machos y $38 \%$ fueron hembras, siendo las proporciones de animales afectados por erliquiosis, similares en ambos grupos. Esta variable ya ha sido estudiada por varios autores encontrando los mismo hallazgos (Contreras et al, 2009; Inokuma et al, 1999), Sin embargo algunos autores consideran que las hembras tienen mayor probabilidad de contraer la enfermedad durante la época de celo, ya que están más expuestas al contacto de varios machos que no siempre tienen un control contra ectoparásitos (Adrianzén et al., 2003).

Se evaluó la variable tiempo de permanencia en casa, teniendo en cuenta que a mayor exposición ambiental existe mayor riesgo de contraer ectoparásitos y por consiguiente aumenta la probabilidad de presentarse enfermedades transmitidas por vectores. El estudio encontró que el grupo de paciente con permanencia exclusiva presentaba menor seropositividad para erliquiosis $(36,4 \%)$ con respecto a los otros grupos. Esto también se ha hallado en otros estudios en los que se reporta que existía diferencia significativa entre los animales que pasaban mayor tiempo a la intemperie con respecto a los que pasaban mayor tiempo en casa (Adrianzén et al., 2003).

En el grupo de animales que tenía permanencia exclusiva en casa, se encontró que el 38\% de ellos tenían garrapatas. Esto quiere decir que a pesar de que los perros no se exponen al medio externo a la casa, se mantiene el riesgo de exposición a garrapatas que se encuentran en el ambiente. Una explicación a este fenómeno podría estar relacionado a que la mayoría de hogares en Piura poseen un jardín mediano o un parque grande cerca de ellas, los cuales son abonados con excremento adquiridos de los establos bovinos y equinos; este abono traería los diferentes estadíos de los ectoparásitos de los perros que viven en estos lugares, lo que puede permitir el contagio a los canes del hogar. Un estudio realizado en la ciudad de Piura, descubrieron Amblyomma maculatum, Ixodes $s p$. Rhipicephalus sanguineus en perros que vivían en establos. Por lo tanto, esto puede indicar que las heces que son extraídas de los establos para abono de parques y jardines podrían llevar consigo garrapatas en varios estadíos (Glenny etal., 2004).

Los signos clínicos encontrados con mayor frecuencia en el estudio fueron debilidad, letargia, membranas mucosas pálidas, anorexia, linfoadenomegalia y esplenomegalia. Los mismos también fueron descritos en un estudio realizado en Yucatán, México (Rodríguez -Vivas, 2004). Sin embargo, a pesar de que uno de los signos clínicos más frecuentes asociado a infecciones por erliquiosis son la uveítis e hifema durante las fases agudas de la enfermedad (Pontes et al., 2004), estos no se reportaron en el estudio.

La presencia serológica de Anaplasma sp. fue reportada por primera vez en la ciudad de Piura por el estudio (4\%). Este porcentaje es similar a lo 
reportado por un estudio realizado en la ciudad de Lima, en donde se halló un $3.3 \%$ de seroreactores (Kennedy, 2012). Se encontró que el distrito de 26 de octubre cuenta con la mayor cantidad de individuos que presentaron anticuerpos para Anaplasma sp. ( $2 / 3$ individuos), mientras que en Piura se halló 1 individuo que presentaba anticuerpos para Anaplasma $s p$. Es importante evaluar con mayor profundidad el comportamiento epidemiológico del Anaplasma sp. en la región, teniendo en consideración que otro estudio realizado en un periodo temporal similar, reporta por primera vez la presencia molecular de A.platys en la región (Cerro, 2017).

Se consideró importante estudiar los hallazgos hematológicos debido a que estas enfermedades alteran de manera frecuente los valores en este examen. (Hoyos et al., 2007). El 33\% de los individuos muestreados positivos a Erlichia spp. mostraron cuadros de bicitopenia (anemia y trombocitopenia). Este hallazgo también ha sido notado en otro estudio, el cual indicaba que estos resultados son importantes para el diagnóstico hematológico, ya que un animal con signos clínicos compatibles a erliquiosis canina, y que manifieste trombocitopenia y anemia (bicitopenia), es muy probable que esté cursando con la enfermedad (Hoyos et al., 2007).

La trombocitopenia es uno de los hallazgos más comunes en los animales con serología positiva a Erlichia spp. (54\%). Los mecanismos que conllevan a esta disminución son múltiples (Harrus et al., 1999). Lo importante es notar que no todos los pacientes mostraron esta alteración, lo cual puede estar asociado a que estos animales puedan estar cursando la etapa subclínica de la enfermedad.

En los pacientes que tuvieron serología negativa también se obtuvieron alteraciones en la serie leucocitaria, de manera similar a lo hallado en los pacientes con erliquiosis. Se determinó que un 38\% presentó leucocitosis y un 50\% monocitosis. Esto puede sugerir que los pacientes podrían presentar un proceso inflamatorio crónico debido a otras patologías como piometra, neumonías, problemas articulares, etc. Otras enfermedades de tipo infecciosas también pueden contribuir a este tipo de hallazgos (se hallaron pacientes con cuerpos de inclusión compatibles con distemper), por lo que sería necesario realizar pruebas complementarias para determinar si existen coinfecciones que puedan explicar estos hallazgos. Se debe considerar la posibilidad que alguno de estos pacientes pueden estar también infectados con la bacteria Ehrlichia spp. y que la serología haya salido negativa, esta reportado que en algunos casos se pueden obtener resultados falsos negativos debido principalmente a una baja seroconversión (Cépeda \& Zapata, 2013; Sánchez, 2016).

El estudio de frotis sanguíneo permitió detectar que dos pacientes presentaron formas compatibles con mórulas de Ehrlichia sp en serie monocito macrófago, lo cual equivale al $5 \%$ del total de animales positivos por serología, lo que estaría indicando la baja sensibilidad de esta técnica en el diagnóstico de la erliquiosis. Otros reportes han demostrado también la baja sensibilidad de esta herramienta, debido a que el tiempo de formación de estas es de 12-17 días y solo suelen hallarse durante la infección aguda (Mylonakis et al., 2003).

\section{CONCLUSIONES}

El estudio realizado llega a las siguientes conclusiones:

Se corrobora que la erliquiosis canina es una enfermedad frecuente $(55 \%)$ en perros con hallazgos de garrapatas en la ciudad de Piura

Se reporta por primera vez una frecuencia serológica positiva a Anaplasma sp. en la ciudad de Piura.

Se encontró una asociación significativa entre las variables permanencia temporal y nula en casa con la presencia de anticuerpos contra E. canis, por lo tanto, es necesario realizar un mejor control de ectoparásitos en los caninos, por medio de antiparasitarios externos en los canes y desinfección del medio ambiente de estos.

\section{Correspondencia:}

Natalia Teresa Naranjo Hurtado

Correo electrónico: natalia.naranjo.h@upch.pe

\section{REFERENCIAS BIBLIOGRAFICAS}

1. Adrianzén, J., Chávez, A., Casas, E., \& Li, O. (2003). Seroprevalencia de la dirofilariosis y ehrlichiosis canina en tres distritos de Lima. Rev Inv Vet, Perú, 14(1), 43-48.

2. Alleman, R., \& Wamsley, H. (2008). An update on anaplasmosis in dogs. dvm360. http:// veterinarymedicine.dvm $360 . \mathrm{com} / \mathrm{update-}$ 
anaplasmosis-dogs

3. Baneth, G. (2006). Canine Ehrlichiosis: A Silent Killer. World Small Animal Veterinary Association World Congress Proceedings. https://www.vin.com/apputil/content/defaultadv1. aspx id $=3859053 \&$ pid $=11223$

4. Chavera, A., Viera, F., \& Samamé, H. (1982). Ehrlichiosis Canina en el Perú. Anales del VII Congreso Nacional de Ciencias Veterinarias, Ica Perú.

5. Cépeda O., \& Zapata, J. (2013). Detección Serológica por Elisa indirecta de hemoparásitos y dirofilaria immitis en caninos de Bogotá. [Tesis de Pregrado]. Universidad La Salle.

6. Cerro, L. (2017). Detecção molecular de Ehrlichia spp., Anaplasma spp., Rickettsia spp. e agentes da Ordem Piroplasmida em cães, e seus carrapatos, da região metropolitana da Província de Piura, Piura-Peru. [Tesis Doctorado]. Universidade Federal Fluminense.

7. Contreras, A., Gavidia, C., Li, O., Díaz, D., \&Hoyos, L. (2009). Estudio retrospectivo de caso-control de ehrlichiosis canina en la Facultad de Medicina Veterinaria de la Universidad Nacional Mayor de San Marcos: Periodo 2002-2005. Rev Investig Vet. Perú, $20(2): 1-53$.

8. Cowell, R., Tyler R., \& Meinkoth J. (1999). Citología y Hematología Diagnóstica en el perro y el gato. Gráfica en Multimedia.

9. Dantas-Torres, F. (2008) Canine vector-borne diseases in Brazil. Parasites Vectors, 1, 25.

10. Dolz, G., Ábrego, L., Romero, L., Campos-Calderón, L., Bouza-Mora, L., \& Jiménez, A. (2013). Ehrlichiosis y anaplasmosis en Costa Rica. Acta Medica Costarricense, S1. http:/www.scielo.sa.cr/ pdf/amc/v55s1/art08.pdf

11. Glenny, M., Mendoza. L\& Falconí, E. (2004). Detección de anticuerpos contra Borrelia burgdorferi e identificación de garrapatas ixodidas en Piura Y Amazonas, Perú. Revista Peruana de Medicina Experimental y Salud Publica, 20 (1).

12. Gordillo, E. (2010). Manual práctico de toma y manejo de muestras en perros y gatos [Tesis Bachiller]. Universidad Veracruzana.

13. Greene, C. (2012). Infectious diseases of the dog and cat. Editorial Elsevier.

14. Hoyos L., Li O., Alvarado A., Suárez F., \& Díaz D. (2007). Evaluación del examen hematológico en el diagnóstico de ehrlichiosis canina. Lima, Perú. Rev. Investig.vet. Perú, 18(2).

15. Huerta, E. \& Dámaso, B. (2015). Factores asociados a la infección por Ehrlichia canis en perros infestados con garrapatas en la ciudad de Huánuco, Perú. Rev Peru Med Exp Salud Publica, 32(4), 756-760.

16. IDEXX laboratorios. (2008). Manual del operador del analizador hematológico IDEXX VetAutoread.
IDEXX laboratorios

17. Inokuma, H., Ohono, K., \&Yamamoto, S. (1999). Serosurvey of Ehrlichia canis y Hepatozoon canis infection in dogs in Yamaguchi Prefecture, Japan. J Vet Med Sci, 61, 1153-1155.

18. Manzano-Román, R., Díaz, V., \& Pérez, R. (2012). Garrapatas: Características anatómicas, epidemiológicas y ciclo vital. Detalles de la influencia de las garrapatas sobre la producción y sanidad animal. Instituto de Recursos Naturales y Agrobiología de Salamanca.

19. Miró, G., Montoya, A., \& Roura, X. (2013) Seropositivity rates for agents of canine vectorborne diseases in Spain: a multicentre study. Parasites Vectors, 6, 117.

20. Mylonakis, M.E., Koutinas, C., Billinis, L.S., Leontides, V., Kontos, O, Papadopoulos, T., \& Fytianou, A. (2003). Evaluation of cytology in the diagnosis of acute canine monocytic ehrlichiosis (Ehrlichia canis): a comparison between five methods. Vet. Microbiol. 91,197- 204

21. Ortega P. (2005). Frecuencia y alteraciones hematológicas asociadas a Ehrlichia spp. en perros domésticos en la ciudad de Cardel, Veracruz, México. [Tesis de Médico Veterinario Zootecnista]. Universidad Veracruzana.

22. Pérez-Écija, R.A., Estepa J.C., \& Mendoza, F.J (2011). Análisis y estudio del frotis sanguíneo. Portal Veterinaria. https://www.portalveterinaria.com/ animales-de-compania/articulos/21842/analisis-yestudio-del-frotis-sanguineo.html

23. Pontes, A., Mendes, P., \& Luiz, J. (2004). Uveitis in dogs infected with Ehrlichia canis. Rev Ciencia Rural Santa María, 34 (4), 1289-1295.

24. Reyes, P. (2003). Determinación de Hemoparásitos de canidos en la zona conurbada Veracruz-Boca del Rio, durante el periodo 1999-2002. [Tesis de Médico Veterinario Zootecnista]. Universidad Veracruzana.

25. Rodríguez-Vivas, R., Albornoz, R., \& Bolio, G. (2004). Ehrlichia canis in dogs in Yucatan, Mexico: seroprevalence, prevalence of infection and associated factors. Vet Parasitol, 127, 75-79.

26. Romero, L., Dolz, G., Romero, J., Meneses, A., Jiménez, M., \& Salazar, L. (2010). Evaluación del diagnóstico de Ehrlichia canis mediante frotis sanguíneo y técnica molecular en perros de Costa Rica. Rev Ciencias Veterinarias, 28 (1): 23-36.

27. Romero, V. (2011). Cambios hematológicos en pacientes positivos a erlichiosis canina en la ciudad de Lázaro Cárdenas Michoacán. [Tesis Bachiller]. Universidad Michoacana de San Nicolás de Hidalgo.

28. Rubio, A., Salas, E., \& Gómez, G. (2011). Presencia de anticuerpos contra Borrelia burgdorferi y Anaplasma sp en canes de la ciudad de Lima. Revista de Investigaciones Veterinarias del Perú, 22, 233238 . 
29. Sánchez, A. (2016). Frecuencia y asociación de alteraciones hematológicas según diagnóstico presuntivo en pacientes caninos atendidos en la Clínica Veterinaria Cayetano Heredia en el período 2013. [Tesis Bachiller]. Lima: Facultad de Medicina Veterinaria y Zootecnia, Universidad Peruana Cayetano Heredia.

30. San Miguel, S. (2006). Prevalencia de Ehrlichia canis en caninos de la provincia de Sullana [Tesis Bachiller]. Universidad Alas Peruanas.
31. Tateishi, V., Lí O., Hoyos L., Rivera H., Manchego A., Barrios L., \& More B. (2015). Identificación hematológica y molecular de Anaplasma platys en caninos domésticos de Lima Metropolitana con signos clínicos compatibles con anaplasmosis. Rev Inv Vet Perú, 26, 111-118.

32. Trapp, S., Dagnone, A., Vidotto, O., Freire, R., Amude, A., \& Autran de Morais, S. (2006). Seroepidemiology of canine babesiosis and ehrlichiosis in a hospital population. Veterinary Parasitology Elsevier, 140, 223-230. 